\title{
Optimisation des programmes de surveillance systématique de l'exposition interne
}

\author{
E. DAVESNE ${ }^{1}$, P. CASANOVA ${ }^{2}$, E. CHOJNACKI $^{3}$, F. PAQUET ${ }^{4}$, \\ E. BLANCHARDON ${ }^{1}$
}

(Manuscrit reçu le 4 janvier 2011, accepté le 15 mars 2011)

RÉSUMÉ En vue d'optimiser la protection des travailleurs vis-à-vis des rayonnements ionisants, la réglementation française impose des limites de dose et une démarche de réduction progressive de l'exposition, dans la continuité des recommandations de la Commission internationale de protection radiologique (CIPR). Afin de vérifier le respect des limites et contraintes de dose lorsqu'un risque significatif de contamination interne existe, des programmes de surveillance sont mis en place, comprenant des mesures radiotoxicologiques périodiques. Cependant, des incertitudes dans l'interprétation dosimétrique de ces mesures sont introduites par leurs variabilités propres et par la connaissance incomplète des circonstances de l'exposition. Ces incertitudes ont été prises en compte par des techniques statistiques bayésiennes. La méthodologie développée a été appliquée à l'analyse du programme de surveillance des travailleurs des ateliers de purification du plutonium du site AREVA NC de La Hague. À partir du seuil de décision du comptage nucléaire, une dose minimale détectable (DMD) par ce programme de routine avec un niveau de confiance donné peut être calculée à l'aide du logiciel «Optimisation des programmes de surveillance de la contamination interne» (OPSCI). Cette méthodologie s'avère un support utile à l'optimisation des programmes de surveillance systématique par la recherche du meilleur compromis entre leur sensibilité et leur coût.

ABSTRACT Optimisation of routine monitoring programmes of internal contamination.

To optimise the protection of workers against ionising radiation, French regulation imposes the use of limits and dose constraints and the progressive decrease of exposition, in line with the recommendations of the International Commission on Radiological Protection. To verify the compliance of the means of protection with these values when a risk of internal contamination exists, monitoring programmes based on periodic bioassay measurements are performed. However, uncertainties in the dose evaluation arise from the variability of the activity measurements and from incomplete knowledge of the exposure conditions. These uncertainties were taken into account by means of Bayesian statistics. The developed methodology was applied to the analysis of the monitoring programme of workers purifying plutonium in the AREVA NC La Hague reprocessing plant. From the decision

IRSN - DRPH, Service de Dosimétrie Interne, BP 17, 92262 Fontenay-aux-Roses Cedex, France.

AREVA NC, Établissement de La Hague, Service de Santé au Travail, 50444 Beaumont-Hague Cedex, France.

IRSN, Direction de la Prévention des Accidents Majeurs, Service d'Étude et de Modélisation de l'Incendie, du Corium et du Confinement, BP 3, 13115 Saint-Paul-Lez-Durance Cedex, France.

4 IRSN, Direction Scientifique, BP 3, 13115 Saint-Paul-Lez-Durance Cedex, France. 
threshold of the measurement, the minimum dose detectable (MDD) by the programme with a given confidence level can be calculated through the software OPSCI. It is shown to be a useful support for the optimisation of monitoring programmes by seeking a compromise between their sensitivity and their cost.

Keywords: Radiological protection / internal dosimetry / monitoring programme / uncertainties / plutonium

\section{Introduction}

La surveillance individuelle des contaminations internes susceptibles de résulter d'une exposition professionnelle est réalisée par des examens anthroporadiométriques et par des mesures radiotoxicologiques. Ces données sont interprétées en termes d'activité incorporée et de dose efficace engagée à l'aide de modèles biocinétiques et dosimétriques. Le risque de contamination est quantifié a priori par l'identification des radionucléides présents au poste de travail, de leurs activités, de la fréquence et de la durée d'exposition. Une technique de mesure et une période de surveillance sont déterminées en fonction des caractéristiques de la mesure et de l'exposition. Pour faciliter ces choix, la norme ISO 20553 (ISO, 2006) recommande des programmes de surveillance spécifiques pour certains composés radioactifs, permettant la détection de toute incorporation annuelle induisant une dose efficace engagée sur 50 ans de plus de $1 \mathrm{mSv}$. Des valeurs nominales sont attribuées aux différents paramètres intervenant dans l'évaluation dosimétrique. Malgré ces recommandations, des incertitudes dans l'interprétation dosimétrique des mesures radiotoxicologiques peuvent subsister.

Les mesures d'activités sont sujettes aux incertitudes liées à la statistique de comptage de la radioactivité qui suit une loi de Poisson (type A), mais également à la détermination du bruit de fond, à l'étalonnage et à l'échantillonnage des prélèvements biologiques. Ces incertitudes de type B sont modélisées par une loi lognormale avec un écart-type géométrique appelé scattering factor (SF) (Marsh et al., 2008). De plus, la connaissance des circonstances d'exposition est souvent incomplète notamment concernant la date de contamination, la taille des particules et l'absorption vers le sang d'un aérosol inhalé.

La sensibilité d'un programme de surveillance en termes de dose efficace engagée peut être déterminée en prenant en compte les incertitudes relatives aux conditions d'exposition et à la mesure. La sensibilité est définie ici comme la capacité d'un programme de surveillance à détecter des contaminations correspondantes à des doses faibles. Pour cela, une incorporation et une dose minimales détectables par le programme de surveillance sont définies à partir du seuil de décision et de la fréquence de la mesure et en fonction des modèles biocinétiques et dosimétriques appliqués. La dose minimale détectable (DMD) est 
définie de telle manière que si la mesure est inférieure au seuil de décision, la dose efficace correspondante est inférieure à la DMD avec un niveau de confiance de $95 \%$. Elle est ainsi un critère de sensibilité du programme de surveillance et peut être utilisée comme un outil d'aide au choix du programme le plus pertinent pour une exposition donnée, en procédant à un compromis entre le coût économique et social des mesures, et la sensibilité de la surveillance.

Le calcul de la DMD relative à une exposition a été réalisé pour les postes de travail des installations d'extraction du plutonium sur le site AREVA NC de La Hague et a permis l'optimisation du programme de surveillance systématique des salariés.

\section{Méthodes de propagation des incertitudes}

La DMD est définie ici comme étant le $95^{\mathrm{e}}$ percentile de la distribution de probabilité de la dose efficace engagée suite aux incorporations de l'année, pour une ou plusieurs mesure(s) observée(s) inférieure(s) ou égale(s) au seuil de décision et compte-tenu des incertitudes sur l'évaluation dosimétrique. Les incertitudes métrologiques et celles liées aux circonstances d'exposition, modélisées par des probabilités, sont propagées pour calculer la distribution de probabilité de l'incorporation et de la dose sachant que la mesure est inférieure ou égale au seuil de décision.

Cette propagation des incertitudes a été réalisée par l'application du théorème de Bayes. La connaissance a priori de la probabilité de l'incorporation est actualisée en une connaissance a posteriori des probabilités de l'incorporation et de la dose après l'observation d'une valeur de mesure $M$ :

$$
\begin{aligned}
P(i \mid M)= & \frac{\iint P(M \mid i, L, t) \times P(L) \times P(t) \times d L \times d t}{\iiint_{L t} P(M \mid i, L, t) \times P(i) \times P(L) \times P(t) \times d L \times d t \times d i} \times P(i), \\
P(E \mid M)= & \frac{\iiint_{L t} P(M \mid E, L, t) \times P(L) \times P(t) \times d L \times d t}{\iiint_{i} P(M \mid E, L, t) \times P(i) \times P(L) \times P(t) \times d L \times d t \times d i} \times P(i) \\
& \text { avec } E=i \times e_{50}(L)
\end{aligned}
$$


$P(i), P(L)$ et $P(t)$ sont les probabilités a priori de l'incorporation $i$, des propriétés physico-chimiques de l'aérosol $L$ et de la date de contamination $t$ : elles représentent l'information connue ou supposée relative à ces variables préalablement à toute mesure. $P(M \mid i, L, t)$ est la vraisemblance de la mesure sachant $i, L$ et $t$, c'est-à-dire la probabilité d'obtenir la mesure $M$ à partir de valeurs données de $i, L$ et $t$. $P(i \mid M)$ t $P(E \mid M)$ sont les probabilités a posteriori de l'incorporation $i$ et de la dose efficace $E$. Elles sont la mise à jour des probabilités a priori par la connaissance apportée par la mesure. Le passage de l'incorporation à la dose efficace $E$ est réalisé en multipliant l'incorporation $i$ par la dose efficace engagée sur 50 ans par unité d'incorporation $e_{50}(L)$ obtenu pour les propriétés physico-chimiques de l'aérosol $L$ données. Le théorème de Bayes peut être appliqué à des variables discrètes en remplaçant les intégrales par des sommes.

Une première méthode, dite Weighted Likelihood Monte-Carlo Sampling (WeLMoS), développée par la Health Protection Agency (HPA, Royaume-Uni ; Puncher and Birchall, 2008) a été appliquée. Elle se base sur l'application du théorème de Bayes à des variables continues et sur une méthode Monte-Carlo pour le calcul implicite des intégrales.

Ensuite, un réseau bayésien a été développé dans le cadre de cette étude pour calculer les probabilités a posteriori de l'incorporation et de la dose à partir d'une mesure (Fig. 1). Il se différencie de la méthode WeLMoS par l'utilisation de variables discrètes. Le réseau peut être décrit ainsi :

- La dose $E$ ne dépend directement que des propriétés physico-chimiques du contaminant $L$ et de l'incorporation $i$.

- L'activité mesurée $M$ dépend de la quantité mesurée $S$ et en diffère à cause des incertitudes de comptage : la probabilité $P(M \mid S)$ d'obtenir $M$ sachant $S$ est ainsi modélisée par une distribution de Poisson de moyenne $S$.

- La quantité $S$ est définie par la prévision du modèle biocinétique suivant les valeurs de $i, L$ et $t$, modulée par des incertitudes de type B : la probabilité de $S$ sachant $i, L$ et $t$ suit une distribution lognormale de moyenne géométrique $i \times m(L, t)$, où $m$ est la fonction d'excrétion ou de rétention correspondant à la quantité mesurée, et avec un écart-type géométrique (SF) dépendant de l'échantillonnage des prélèvements et de l'étalonnage du système de mesure.

La vraisemblance globale associée à une valeur d'incorporation ou de dose est obtenue alors en sommant les vraisemblances obtenues pour chaque combinaison des valeurs discrètes des différentes variables. 


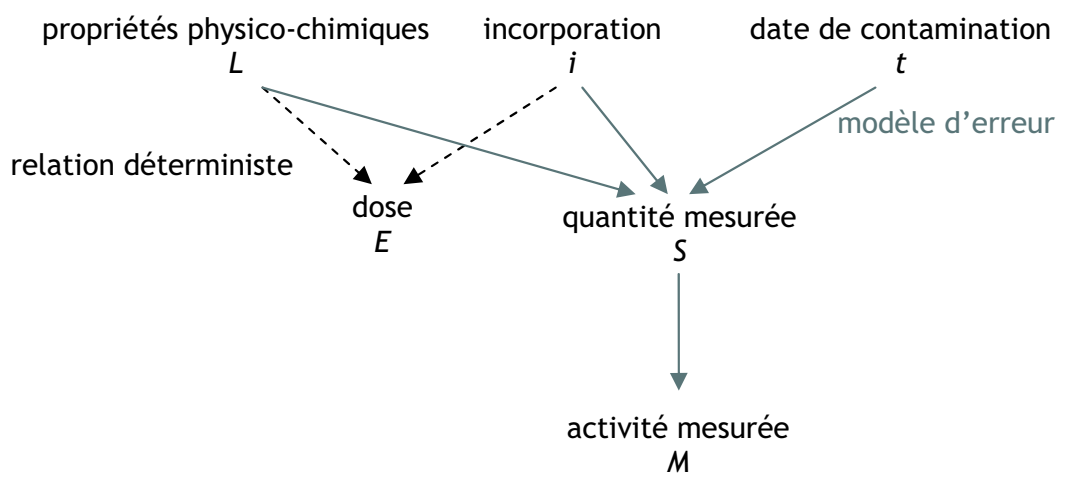

Figure 1 - Structure du réseau bayésien développé pour obtenir les probabilités a posteriori de l'incorporation et de la dose.

Bayesian network developed to calculate the posterior probabilities of the intake and of the dose.

\section{Comparaison entre le réseau bayésien et la méthode WeLMoS}

\subsection{Méthode}

Le réseau bayésien et la méthode $\mathrm{WeLMoS}$ résolvent le même problème avec des hypothèses similaires excepté l'utilisation de variables continues ou discrètes. La comparaison de leurs résultats contribue donc à valider leur implémentation. Une modélisation simple des paramètres a été appliquée à des fins de comparaison :

- contamination par inhalation d'un aérosol de ${ }^{239} \mathrm{Pu}$ avec une absorption pulmonaire de type $\mathrm{S}$ (ICRP, 1994) et un diamètre aérodynamique médian en activité (DAMA) de 1, 5 ou $10 \mu \mathrm{m}$ avec la même probabilité ;

- une mesure fécale de routine égale à 50 coups avec un bruit de fond moyen de 0,1 coup ;

- $\mathrm{SF}=3$;

- probabilité a priori de $i$ uniforme entre 1 et $23 \mathrm{~Bq}$ (pour obtenir 10 valeurs d'i);

- probabilité a priori de $t$ uniforme entre 0 et $180 \mathrm{j}$.

La discrétisation du réseau bayésien est présentée dans le tableau I. Pour la dose, la discrétisation contient les valeurs de dose pour lesquelles la probabilité a posteriori sera calculée. Elle est donc ajustée selon l'utilisation qui sera faite des résultats. Pour cette comparaison, les valeurs échantillonnées de la date de contamination, du DAMA et de l'absorption utilisées par la méthode WeLMoS sont égales aux valeurs discrètes de ces variables pour le réseau bayésien. Les 


\section{TABLEAU I}

Discrétisation des variables dans le réseau bayésien pour la comparaison avec la méthode WeLMoS.

Discretisation of the variables in the Bayesian network for comparison with the WeLMoS method.

\begin{tabular}{cc}
\hline Variable & Discrétisation \\
\hline Incorporation $(\mathrm{Bq})$ & $1 ; 3 ; 5 ; 8 ; 10 ; 13 ; 15 ; 18 ; 20 ; 23$ \\
DAMA $(\mu \mathrm{m})$ & $1 ; 5 ; 10$ \\
Absorption vers le sang & Type $\mathrm{S}$ \\
Date de contamination $(\mathrm{j})$ & $1 ; 20 ; 40 ; 60 ; 90 ; 110 ; 130 ; 150 ; 170 ; 180$ \\
\hline Quantité mesurée $(\mathrm{Bq})$ & $0 ; 10^{-5} ; 10^{-4} ; 2 \times 10^{-4} ; 3 \times 10^{-4} ; 5 \times 10^{-4} ; 7 \times 10^{-4} ; 9 \times 10^{-4} ; 1,1 \times 10^{-3} ;$ \\
& $1,3 \times 10^{-3} ; 1,7 \times 10^{-3} ; 3 \times 10^{-2} ; 5 \times 10^{-2} ; 10^{-1} ; 5 \times 10^{-1} ; 1 ; 3 ; 5 ; 10$ \\
\hline Dose $(\mathrm{mSv})$ & $0 ; 10^{-3} ; 10^{-2} ; 5 \times 10^{-2} ; 0,1 ; 0,15 ; 0,2 ; 0,25 ; 0,3 ; 0,35 ; 0,4 ; 0,45 ; 0,5 ; 0,55 ;$ \\
& 0,$6 ; 0,65$ \\
\hline
\end{tabular}

erreurs de type B sont modélisées par une loi lognormale et intégrée différemment dans les deux méthodes. Pour le réseau, elles sont représentées par la probabilité $P(S \mid i, L, t)$ comme présenté précédemment. Pour la méthode WeLMoS, elles sont intégrées en introduisant un facteur d'erreur multiplicatif $f$ distribué selon une loi lognormale ayant le même écart-type géométrique que celui utilisé pour $P(S \mid i, L$, t) dans le réseau bayésien : SF. Pour cette comparaison, dix valeurs de $f$ ont été échantillonnées par méthode Monte-Carlo en suivant une loi lognormale de moyenne géométrique 1 et pour écart-type géométrique SF.

\subsection{Résultats et discussion}

Les résultats de la comparaison sont représentés dans la figure 2. La méthode WeLMoS évalue les probabilités conditionnelles de l'incorporation ou de la dose sachant la mesure par un modèle numérique continu et par une méthode de MonteCarlo c'est-à-dire par un calcul intégral implicite. Ceci permet d'évaluer précisément des grandeurs statistiques telles que la médiane ou le percentile $95 \%$. Le réseau bayésien évalue les probabilités conditionnelles en utilisant une modélisation numérique discrète et une intégration explicite. De ce fait, le résultat du réseau bayésien associé aux différentes grandeurs statistiques d'intérêt est un intervalle incluant cette grandeur dont la largeur dépend à la fois du problème modélisé et de sa discrétisation numérique.

Les valeurs des médianes et $95^{\mathrm{e}}$ percentiles obtenues avec la méthode WeLMoS sont contenues ou proches des intervalles calculés par le réseau bayésien, indiquant la cohérence des deux méthodes d'autant que le modèle pris pour modéliser l'incertitude de type B n'est pas exactement équivalent. 


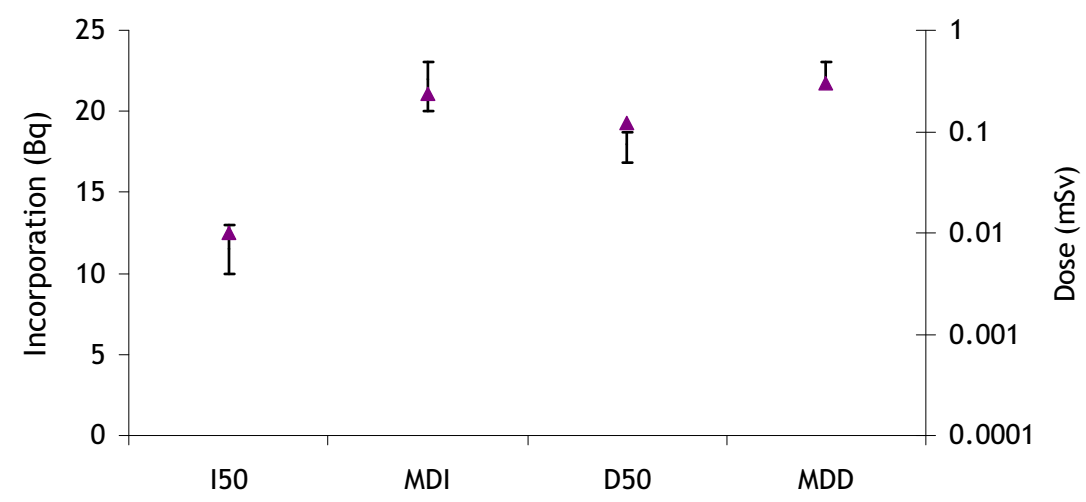

Figure 2 - Comparaison des résultats des méthodes WeLMoS ( $\mathbf{\Delta})$ et du réseau bayésien (intervalles) pour un problème physique identique (description détaillée dans le paragraphe 2.2). I50 (respectivement D50): incorporation (respectivement dose) médiane, MDI (MDD) : $95^{e}$ percentile de l'incorporation (dose) ou incorporation (dose) minimale détectable avec un niveau de confiance de $95 \%$.

Comparison of the results obtained with the WeLMoS method (A) and with the Bayesian network (intervals) for the same problem described in Section 2.2. 150 (respectively D50): median intake (respectively dose); MDI (respectively MDD): minimum detectable intake (respectively dose) with a confidence level of $95 \%$.

La précision numérique relative aux grandeurs statistiques (e.g. percentiles) de la méthode WeLMoS dépend uniquement du nombre de simulations Monte-Carlo effectuées. Ainsi, une solution rapide et efficace est obtenue pour propager les incertitudes si les probabilités des combinaisons de variables sont connues. Cependant, la robustesse des résultats par rapport aux hypothèses prises dans la modélisation des incertitudes par exemple lorsqu'un choix de distribution de probabilité est fait pour la représenter est difficile à estimer parce que le lien entre les poids de probabilité associés aux valeurs prises par les données : «temps d'incorporation », « incorporation »..., et le poids de probabilité associé aux valeurs prises par les résultats : « dose » ou « incorporation » après, est implicite.

En revanche, le réseau bayésien détermine explicitement la probabilité de chaque combinaison de valeurs des paramètres. Ainsi, un changement d'hypothèse sur la modélisation d'un paramètre incertain induit une pondération différente des sommes et son impact peut être évalué directement. Les avantages du réseau bayésien sont donc sa lisibilité et dans l'appréciation de la robustesse face aux choix de modélisation pris pour rendre compte des incertitudes. Cependant, le temps de calcul peut devenir limitant suivant le nombre de paramètres incertains et leur discrétisation. 
La différence entre le réseau bayésien et la méthode WeLMoS se situe dans la transcription numérique du problème. Le réseau bayésien établit de façon explicite le lien entre les poids des probabilités des données et ceux des résultats. Pour ce faire, il est nécessaire d'avoir recours à une discrétisation des variables. Le réseau évalue alors la vraisemblance d'un intervalle entre deux valeurs discrètes. $\mathrm{Au}$ contraire, la méthode WeLMoS évalue les poids de probabilités des résultats en utilisant une technique de Monte-Carlo, ce qui ne nécessite pas de discrétiser les variables incertaines et rend la qualité de la résolution indépendante du nombre de variables considérées. C'est pourquoi, la méthode WeLMoS fournit des résultats d'une grande précision numérique si et seulement si la modélisation des incertitudes est basée sur des données réelles et précises. Pourtant, attribuer des probabilités a priori continues est une hypothèse forte qui peut ne pas être fondée sur des informations spécifiques comme pour la probabilité a priori uniforme attribuée à la date de contamination. En attribuant des poids de probabilités sur les intervalles délimités par la discrétisation, le réseau bayésien est moins exigeant en termes d'hypothèse nécessaires pour modéliser l'incertitude et permet d'évaluer $a$ priori l'impact d'un choix différent de modélisation. En raison de leur complémentarité et pour permettre une comparaison, les deux méthodes sont utilisées dans cette étude.

\section{Application des méthodes}

\subsection{Modélisation de l'exposition potentielle et de la mesure}

Les ateliers d'extraction du plutonium $(\mathrm{Pu})$ sur le centre de retraitement AREVA $\mathrm{NC}$ de La Hague (R4 et T4) reçoivent une solution de nitrate de plutonium provenant de la séparation d'avec l'uranium et les produits de fission à partir du combustible usé. Dans un premier temps, le $\mathrm{Pu}$ est extrait par extraction liquideliquide puis concentré et purifié sous forme de précipité d'oxalate. L'oxalate est ensuite filtré puis séché et placé dans un four pour être oxydé. Le dioxyde de plutonium est successivement homogénéisé puis conditionné dans des boîtes avant entreposage. Différentes compositions isotopiques du $\mathrm{Pu}$ sont rencontrées en raison de la diversité des provenances du combustible retraité : réacteur à eau pressurisée (REP) ou à eau bouillante (REB), combustible MOX irradié seul ou mélangé avec des reliquats de $\mathrm{Pu}$ recyclé.

Grâce à l'automatisation du procédé, les travailleurs restent la plupart du temps en salle de conduite. Une contamination ne pourrait avoir lieu que lors d'interventions en boîte à gant pour le nettoyage hebdomadaire du filtre rotatif, le recyclage une à deux fois par semaine des reliquats de plutonium provenant d'analyse, ou la maintenance en cas de problème particulier comme le mauvais 
placement d'une boîte de conditionnement. En cas de rupture de confinement d'une boîte à gant, les travailleurs sont susceptibles d'inhaler de l'oxalate ou du dioxyde de plutonium insoluble lors de ces opérations. C'est pourquoi leur programme de surveillance repose sur des mesures de spectrométries alpha semestrielles sur des échantillons de selle.

La durée de mesure est de $48 \mathrm{~h}$. Dans la région d'intérêt permettant d'identifier les ${ }^{239} \mathrm{Pu}$ et ${ }^{240} \mathrm{Pu}$ qui ne peuvent être séparés par spectrométrie alpha, le bruit de fond moyen est de 2,2 coups par $48 \mathrm{~h}$. Le seuil de décision de 4,2 coups peut en être déduit pour ces isotopes (Vivier et al., 2010). L'efficacité moyenne de détection des particules alphas par le détecteur est de 32,5\%. Le rendement chimique moyen de préparation des échantillons pour le plutonium est de $86 \%$. Ces données ont permis de représenter précisément le processus de mesures.

Les incertitudes sur les conditions d'exposition et la mesure sont modélisées :

- la date de contamination inconnue pendant l'intervalle de surveillance est représentée par une loi de probabilité uniforme entre 0 et 180 jours avant la mesure fécale semestrielle ;

- l'incertitude sur le diamètre des particules est modélisée par une probabilité uniforme du DAMA entre 1 et $10 \mu \mathrm{m}$ faute d'information plus précise ;

- l'incertitude sur la solubilité du $\mathrm{PuO}_{2}$ est quantifiée en utilisant les valeurs réalistes synthétisées par Davesne et al. (2010) pour le dioxyde de Pu et le MOX ;

- le modèle d'incertitude de mesure est de type Poisson, avec un bruit de fond moyen de 2,2 coups/48 h. L'incertitude de type B suit une loi lognormale d'écart-type géométrique 3 (Marsh et al., 2008).

L'événement minimal détecté par la technique de mesure correspond alors à l'observation de 5 coups/48 h.

Trois hypothèses de probabilité a priori de l'incorporation $P(i)$ ont été considérées: une densité constante $P(i)=1$ suppose que toutes les valeurs d'activité incorporée sont équiprobables entre 0 et $1000 \mathrm{~Bq}$; des densités décroissantes exponentielle $P(i)=e^{-i}$ et $«$ alpha $\gg P(i)=\alpha \cdot i^{a-1}$ avec $\alpha=0,001$ supposent que les incorporations les plus faibles sont les plus vraisemblables. Le paramètre $\alpha$ représente la fréquence de mesures positives pour un groupe de travailleur donné (Miller et al., 2001). Ces probabilités a priori ont été choisies comme des hypothèses extrêmes pouvant être faites avant toute mesure. 
TABLEAU II

Discrétisation optimale des variables dans le réseau bayésien pour le calcul de la DMD. Optimum discretisation of the variables in the Bayesian network to calculate the MDD.

\begin{tabular}{cc}
\hline Variable & Discrétisation \\
\hline Incorporation $(\mathrm{Bq})$ & $0 ; 10^{-4} ; 5 \times 10^{-4} ; 10^{-3} ; 5 \times 10^{-3} ; 10^{-2} ; 5 \times 10^{-2} ; 10^{-1} ; 5 \times 10^{-1} ; 1 ; 5 ; 10 ; 20 ;$ \\
$30 ; 40 ; 50 ; 100 ; 150 ; 300 ; 600 ; 1000$ & $1 ; 3 ; 5 ; 10$ \\
DAMA $(\mu \mathrm{m})$ & Type M $;$ Type S \\
Absorption vers le sang & $170 ; 180$ \\
Date de contamination $(\mathrm{j})$ & $0 ; 10 ; 20 ; 30 ; 40 ; 50 ; 60 ; 70 ; 80 ; 90 ; 100 ; 110 ; 120 ; 130 ; 140 ; 150 ; 160 ;$ \\
& \\
Quantité mesurée $(\mathrm{Bq})$ & $0 ; 10^{-6} ; 10^{-5} ; 10^{-4} ; 5 \times 10^{-4} ; 10^{-3} ; 5 \times 10^{-3} ; 10^{-2} ; 5 \times 10^{-2} ; 10^{-1} ; 5 \times 10^{-1} ; 1 ;$ \\
& $5 ; 10 ; 50 ; 100 ; 200 ; 500 ; 1000$ \\
Dose $(\mathrm{mSv})$ & $0 ; 10^{-3} ; 10^{-2} ; 10^{-1} ; 5 \times 10^{-1} ; 1 ; 2 ; 3 ; 4 ; 5 ; 6 ; 7 ; 8 ; 9 ; 10 ; 15 ; 20 ; 50 ; 100 ;$ \\
& $500 ; 1000 ; 10000$ \\
\hline
\end{tabular}

La discrétisation du réseau bayésien a été déterminée afin d'optimiser le temps de calcul (Tab. II). Les discrétisations de l'incorporation et de la dose sont ajustées selon la distribution de probabilité recherchée comme résultat. Le jeu de 66 valeurs des paramètres d'absorption pulmonaires dérivées de Davesne et al. (2010) pour le dioxyde de Pu et le MOX est trop grand pour un temps de calcul raisonnable. C'est pourquoi, au sein du réseau, l'absorption est modélisée comme un mélange de types $M$ et $S$ (ICRP, 1994) avec des probabilités de 0,162 et 0,838 respectivement obtenues à partir de la distribution des valeurs du coefficient de dose calculées par Davesne et al. (2010). Pour le DAMA, la discrétisation du CDROM de la CIPR (ICRP, 1998) a été utilisée.

\subsection{Dose minimale détectable pour les ateliers Pu du site AREVA NC La Hague}

La DMD annuelle est calculée en prenant en compte l'ensemble des mesures de routine réalisées dans l'année, prises inférieures ou égales au seuil de décision. Les résultats sont présentés dans le tableau III pour les différents combustibles. La DMD a été calculée en considérant les isotopes ${ }^{239} \mathrm{Pu}$ et ${ }^{240} \mathrm{Pu}$ comme les traceurs permettant de remonter à la dose correspondant à l'ensemble du plutonium.

Les différences entre les DMD calculées par la méthode WeLMoS et le réseau bayésien sont induites par la définition du problème physique. En effet, pour la méthode WeLMoS, les probabilités a priori sont continues pour tous les paramètres incertains sauf l'absorption vers le sang qui est modélisée par les résultats de Davesne et al. (2010). Pour le réseau bayésien, les paramètres incertains ainsi que leurs probabilités a priori sont discrétisées et l'absorption vers le sang est modélisée par un mélange de types $\mathrm{M}$ et $\mathrm{S}$. Cette étude montre donc 


\section{TABLEAU III}

DMD annuelles calculées par la méthode WeLMoS et le réseau bayésien avec des probabilités $a$ priori de l'incorporation uniforme, exponentielle, ou alpha. Application aux travailleurs de R4T4 pour une mesure fécale inférieure ou égale au seuil de décision pour différents combustibles (réacteurs à eau pressurisée REP1 et REP2, à eau bouillante REB, MOX irradié seul ou mélangé avec du Pu recyclé MOX_RP).

Annual MDDs obtained by the WeLMoS method and by the Bayesian network with uniform, exponential or alpha intake prior probability. Application to workers of R4-T4 facilities for observed faecal measurements below or at the decision threshold for different spent fuels (pressurized water reactor REP1 and REP2, boiling water reactor REB, MOX alone or mixed with recycled Pu MOX_RP).

\begin{tabular}{|c|c|c|c|}
\hline $\begin{array}{c}\text { Probabilité a priori de } \\
\text { l'incorporation }\end{array}$ & Méthode & Combustible & DMD $(\mathbf{m S v})$ \\
\hline \multirow{10}{*}{$P(i)$ uniforme } & \multirow{5}{*}{ WeLMoS } & REP1 & 1,7 \\
\hline & & REP2 & 1,8 \\
\hline & & REB & 1,8 \\
\hline & & MOX & 1,8 \\
\hline & & MOX_RP & 1,8 \\
\hline & \multirow{5}{*}{ Réseau bayésien } & REP1 & $1<\mathrm{DMD}<2$ \\
\hline & & REP2 & $1<\mathrm{DMD}<2$ \\
\hline & & REB & $1<\mathrm{DMD}<2$ \\
\hline & & MOX & $1<\mathrm{DMD}<2$ \\
\hline & & MOX_RP & $0,5<\mathrm{DMD}<1$ \\
\hline \multirow{10}{*}{$P(i)$ exponentielle } & \multirow{5}{*}{ WeLMoS } & REP1 & $1,6 \times 10^{-1}$ \\
\hline & & REP2 & $1,7 \times 10^{-1}$ \\
\hline & & REB & $1,6 \times 10^{-1}$ \\
\hline & & MOX & $1,6 \times 10^{-1}$ \\
\hline & & MOX_RP & $1,5 \times 10^{-1}$ \\
\hline & \multirow{5}{*}{ Réseau bayésien } & REP1 & $0,01<\mathrm{DMD}<0,1$ \\
\hline & & REP2 & $0,01<\mathrm{DMD}<0,1$ \\
\hline & & REB & $0,01<\mathrm{DMD}<0,1$ \\
\hline & & MOX & $0,01<\mathrm{DMD}<0,1$ \\
\hline & & MOX_RP & $0,01<\mathrm{DMD}<0,1$ \\
\hline \multirow{10}{*}{$P(i)$ alpha } & \multirow{5}{*}{ WeLMoS } & REP1 & $\mathrm{DMD}<10^{-6}$ \\
\hline & & REP2 & $\mathrm{DMD}<10^{-6}$ \\
\hline & & REB & $\mathrm{DMD}<10^{-6}$ \\
\hline & & MOX & $\mathrm{DMD}<10^{-6}$ \\
\hline & & MOX_RP & $\mathrm{DMD}<10^{-6}$ \\
\hline & \multirow{5}{*}{ Réseau bayésien } & REP1 & $\mathrm{DMD}=0^{\mathrm{a}}$ \\
\hline & & REP2 & $\mathrm{DMD}=0^{\mathrm{a}}$ \\
\hline & & REB & $\mathrm{DMD}=0^{\mathrm{a}}$ \\
\hline & & MOX & $\mathrm{DMD}=0^{\mathrm{a}}$ \\
\hline & & MOX_RP & $\mathrm{DMD}=0^{\mathrm{a}}$ \\
\hline
\end{tabular}

\footnotetext{
${ }^{\mathrm{a}} \mathrm{La}$ dose $0 \mathrm{mSv}$ correspond à un percentile supérieur au $95^{\mathrm{e}}$.
} 
l'influence de la modélisation du problème (choix des lois de probabilité pour modéliser les incertitudes) et choix de leur transcription numérique (intégration implicite ou explicite) sur le résultat. De plus, cette comparaison permet de valider les résultats de l'optimisation car les deux méthodes fournissent des DMD similaires.

D'autre part, une étude de sensibilité a été réalisée pour déterminer quels paramètres influent le plus sur l'évaluation de la DMD. Elle a montré que la probabilité a priori de l'incorporation et la solubilité du composé sont les plus influents. En revanche la variation du DAMA a peu d'effet sur le résultat.

\subsection{Optimisation du programme de surveillance}

L'exploitant veut assurer la détection d'incorporation correspondant à une dose efficace annuelle de $1 \mathrm{mSv}$. Ainsi, après avoir évalué le programme de surveillance en place par le calcul de la DMD, les DMD de différents programmes envisageables ont été calculées :

(a) une mesure fécale tous les 2 ans,

(b) une mesure fécale tous les ans,

(c) une mesure fécale tous les 6 mois,

(d) une mesure urinaire tous les 6 mois,

(e) une mesure urinaire annuelle suivie d'une mesure fécale 6 mois après,

(f) une mesure fécale annuelle suivie d'une mesure urinaire 6 mois après,

(g) une mesure urinaire et une mesure fécale simultanées tous les 6 mois,

(h) une mesure fécale tous les 3 mois,

(i) une mesure urinaire tous les 3 mois.

Trois réseaux bayésiens ont été utilisés et comprennent 1, 2 ou 4 mesures. Leurs discrétisations sont présentées dans le tableau IV.

Les résultats sont regroupés dans le tableau V. En se basant sur $P(i)$ uniforme comme probabilité a priori de l'incorporation, seuls les programmes de surveillance $\mathrm{g}$ et h permettent d'assurer que la DMD est inférieure à $1 \mathrm{mSv}$. Pour $P(i)=e^{-i}$, tous les programmes envisagés permettent de détecter une contamination menant à une dose de $1 \mathrm{mSv}$. Les programmes $\mathrm{g}$ et $\mathrm{h}$ sont formés de quatre mesures par an ce qui est coûteux financièrement et contraignant en pratique. De plus, supposer que toutes les incorporations entre 1 et $1000 \mathrm{~Bq}$ ont la même probabilité de se produire sans être détectées par la surveillance d'ambiance est très prudent pour cette installation où seulement une mesure fécale sur 1000 est positive. 
TABLEAU IV

Discrétisations des variables dans le réseau bayésien.

Discretisation of the variables in the Bayesian network.

\begin{tabular}{|c|c|c|}
\hline $\begin{array}{l}\text { Nombres de } \\
\text { mesures par an }\end{array}$ & Variable & Discrétisation \\
\hline \multirow{6}{*}{1} & Incorporation $(\mathrm{Bq})$ & $\begin{array}{c}0 ; 10^{-4} ; 5 \times 10^{-4} ; 10^{-3} ; 5 \times 10^{-3} ; 10^{-2} ; 5 \times 10^{-2} ; 10^{-1} ; 5 \times \\
10^{-1} ; 1,0 ; 5 ; 10 ; 20 ; 30 ; 40 ; 50 ; 100 ; 150 ; 300 ; 600 ; 1000\end{array}$ \\
\hline & DAMA $(\mu \mathrm{m})$ & $1 ; 3 ; 5 ; 10$ \\
\hline & Absorption pulmonaire & Type M ; Type S \\
\hline & Date de contamination $(\mathrm{j})$ & $\begin{array}{c}0 ; 10 ; 20 ; 30 ; 40 ; 50 ; 60 ; 70 ; 80 ; 90 ; 100 ; 110 ; 120 ; 130 ; \\
140 ; 150 ; 160 ; 170 ; 180\end{array}$ \\
\hline & Quantité mesurée (Bq) & $\begin{array}{c}0 ; 10^{-7} ; 10^{-6} ; 5 \times 10^{-6} ; 10^{-5} ; 2 \times 10^{-5} ; 3 \times 10^{-5} ; 4 \times 10^{-5} ; \\
5 \times 10^{-5} ; 6 \times 10^{-5} ; 7 \times 10^{-5} ; 8 \times 10^{-5} ; 9 \times 10^{-5} ; 10^{-4} ; 2 \times \\
10^{-4} ; 3 \times 10^{-4} ; 4 \times 10^{-4} ; 5 \times 10^{-4} ; 6 \times 10^{-4} ; 7 \times 10^{-4} ; 8 \times \\
10^{-4} ; 9 \times 10^{-4} ; 10^{-3} ; 2 \times 10^{-3} ; 3 \times 10^{-3} ; 4 \times 10^{-3} ; 5 \times 10^{-3} ; \\
6 \times 10^{-3} ; 7 \times 10^{-3} ; 8 \times 10^{-3} ; 9 \times 10^{-3} ; 10^{-2} ; 2 \times 10^{-2} ; 3 \times \\
10^{-2} ; 4 \times 10^{-2} ; 5 \times 10^{-2} ; 6 \times 10^{-2} ; 7 \times 10^{-2} ; 8 \times 10^{-2} ; 9 \times \\
10^{-2} ; 10^{-1} ; 2 \times 10^{-1} ; 3 \times 10^{-1} ; 4 \times 10^{-1} ; 5 \times 10^{-1} ; 6 \times 10^{-1} ; \\
7 \times 10^{-1} ; 8 \times 10^{-1} ; 9 \times 10^{-1} ; 1 ; 2 ; 3 ; 4 ; 5 ; 6 ; 7 ; 8 ; 9 ; 10 ; \\
20 ; 30 ; 40 ; 50 ; 60 ; 70 ; 80 ; 90 ; 100 ; 200 ; 300 ; 400 ; 500 ; \\
600 ; 700 ; 800 ; 900 ; 1000\end{array}$ \\
\hline & Dose $(\mathrm{mSv})$ & $\begin{array}{c}0 ; 10^{-3} ; 10^{-2} ; 10^{-1} ; 5 \times 10^{-1} ; 1 ; 2 ; 3 ; 4 ; 5 ; 6 ; 7 ; 8 ; 9 ; 10 ; \\
15 ; 20 ; 50 ; 100 ; 500 ; 1000 ; 10000\end{array}$ \\
\hline \multirow{6}{*}{2} & Incorporation $(\mathrm{Bq})$ & $\begin{array}{l}0 ; 10^{-4} ; 5 \times 10^{-4} ; 10^{-3} ; 5 \times 10^{-3} ; 10^{-2} ; 5 \times 10^{-2} ; 10^{-1} ; 5 \times \\
10^{-1} ; 1 ; 5 ; 10 ; 20 ; 30 ; 40 ; 50 ; 100 ; 150 ; 300 ; 600 ; 1000\end{array}$ \\
\hline & DAMA $(\mu \mathrm{m})$ & $1 ; 3 ; 5 ; 10$ \\
\hline & Absorption pulmonaire & Type M ; Type S \\
\hline & Date de contamination (j) & $\begin{array}{c}0 ; 10 ; 20 ; 30 ; 40 ; 50 ; 60 ; 70 ; 80 ; 90 ; 100 ; 110 ; 120 ; 130 ; \\
140 ; 150 ; 160 ; 170 ; 180\end{array}$ \\
\hline & Quantité mesurée (Bq) & $\begin{array}{c}0 ; 10^{-6} ; 10^{-5} ; 10^{-4} ; 5 \times 10^{-4} ; 10^{-3} ; 5 \times 10^{-3} ; 10^{-2} ; 5 \times 10^{-2} ; \\
10^{-1} ; 5 \times 10^{-1} ; 1 ; 5 ; 10 ; 50 ; 100 ; 200 ; 500 ; 1000\end{array}$ \\
\hline & Dose $(\mathrm{mSv})$ & $\begin{array}{c}0 ; 10^{-3} ; 10^{-2} ; 10^{-1} ; 5 \times 10^{-1} ; 1 ; 2 ; 3 ; 4 ; 5 ; 6 ; 7 ; 8 ; 9 ; 10 ; \\
15 ; 20 ; 50 ; 100 ; 500 ; 1000 ; 10000\end{array}$ \\
\hline \multirow{6}{*}{4} & Incorporation $(\mathrm{Bq})$ & $0 ; 10^{-4} ; 10^{-3} ; 10^{-2} ; 10^{-1} ; 1 ; 10 ; 100 ; 1000$ \\
\hline & DAMA $(\mu \mathrm{m})$ & $1 ; 5 ; 10$ \\
\hline & Absorption pulmonaire & Type M ; Type S \\
\hline & Date de contamination (j) & $0 ; 10 ; 90 ; 180$ \\
\hline & Quantité mesurée (Bq) & $\begin{array}{c}0 ; 10^{-6} ; 10^{-5} ; 10^{-4} ; 5 \times 10^{-4} ; 10^{-3} ; 5 \times 10^{-3} ; 10^{-2} ; 5 \times 10^{-2} ; \\
10^{-1} ; 5 \times 10^{-1} ; 1 ; 5 ; 10 ; 50 ; 100 ; 1000\end{array}$ \\
\hline & Dose $(\mathrm{mSv})$ & $0 ; 10^{-3} ; 10^{-1} ; 5 \times 10^{-1} ; 1 ; 5 ; 10 ; 20 ; 1000 ; 10000$ \\
\hline
\end{tabular}




\section{TABLEAU V}

DMD calculées pour les différents programmes de surveillance en utilisant le réseau bayésien avec la modélisation et la discrétisation présentées dans le texte. Application aux travailleurs de R4-T4 pour une mesure fécale inférieure ou égale au seuil de décision et différents combustibles usagés (réacteurs à eau pressurisée REP1 et REP2, à eau bouillante REB, MOX irradié seul ou mélangé avec du Pu recyclé MOX_RP).

MDDs calculated for different monitoring programmes using the Bayesian network with parameters modelling and discretisations presented in text. Application to workers of R4-T4 facilities for observed faecal measurements below or at the decision threshold and different spent fuels (pressurized water reactor REP1 and REP2, boiling water reactor REB, MOX alone or mixed with recycled Pu MOX_RP).

\begin{tabular}{|c|c|c|c|c|}
\hline \multirow{2}{*}{$\begin{array}{l}\text { Composition } \\
\text { isotopique }\end{array}$} & \multicolumn{4}{|c|}{ Dose minimale détectable avec un niveau de confiance de $95 \%(\mathrm{mSv})$} \\
\hline & Programme & $P(i)$ uniforme & $P(i)$ exponentielle & $P(i)$ alpha \\
\hline \multirow{9}{*}{ REP1 } & $\mathrm{a}$ & $20<\mathrm{DMD}<50$ & $0,1<\mathrm{DMD}<0,5$ & $\mathrm{DMD}=0^{\mathrm{a}}$ \\
\hline & $\mathrm{b}$ & $10<\mathrm{DMD}<15$ & $0,1<\mathrm{DMD}<0,5$ & $\mathrm{DMD}=0^{\mathrm{a}}$ \\
\hline & $\mathrm{c}$ & $1<\mathrm{DMD}<2$ & $0,01<\mathrm{DMD}<0,1$ & $\mathrm{DMD}=0^{\mathrm{a}}$ \\
\hline & $\mathrm{d}$ & $20<\mathrm{DMD}<50$ & $0,1<\mathrm{DMD}<0,5$ & $\mathrm{DMD}=0^{\mathrm{a}}$ \\
\hline & $\mathrm{e}$ & $1<\mathrm{DMD}<2$ & $0,01<\mathrm{DMD}<0,1$ & $\mathrm{DMD}=0^{\mathrm{a}}$ \\
\hline & $\mathrm{f}$ & $2<\mathrm{DMD}<3$ & $0,1<\mathrm{DMD}<0,5$ & $\mathrm{DMD}=0^{\mathrm{a}}$ \\
\hline & $\mathrm{g}$ & $0,1<\mathrm{DMD}<0,5$ & $0,001<\mathrm{DMD}<0,1$ & $\mathrm{DMD}=0^{\mathrm{a}}$ \\
\hline & $\mathrm{h}$ & $0,001<\mathrm{DMD}<0,1$ & $0,001<\mathrm{DMD}<0,1$ & $\mathrm{DMD}=0^{\mathrm{a}}$ \\
\hline & $\mathrm{i}$ & $1<\mathrm{DMD}<5$ & $0,5<\mathrm{DMD}<1$ & $\mathrm{DMD}=0^{\mathrm{a}}$ \\
\hline \multirow{9}{*}{ REP2 } & a & $20<\mathrm{DMD}<50$ & $0,1<\mathrm{DMD}<0,5$ & $\mathrm{DMD}=0^{\mathrm{a}}$ \\
\hline & $\mathrm{b}$ & $15<\mathrm{DMD}<20$ & $0,1<\mathrm{DMD}<0,5$ & $\mathrm{DMD}=0^{\mathrm{a}}$ \\
\hline & c & $1<\mathrm{DMD}<2$ & $0,01<\mathrm{DMD}<0,1$ & $\mathrm{DMD}=0^{\mathrm{a}}$ \\
\hline & d & $20<\mathrm{DMD}<50$ & $0,1<\mathrm{DMD}<0,5$ & $\mathrm{DMD}=0^{\mathrm{a}}$ \\
\hline & e & $1<\mathrm{DMD}<2$ & $0,1<\mathrm{DMD}<0,5$ & $\mathrm{DMD}=0^{\mathrm{a}}$ \\
\hline & $\mathrm{f}$ & $3<\mathrm{DMD}<4$ & $0,1<\mathrm{DMD}<0,5$ & $\mathrm{DMD}=0^{\mathrm{a}}$ \\
\hline & $\mathrm{g}$ & $0,1<\mathrm{DMD}<0,5$ & $0,001<\mathrm{DMD}<0,1$ & $\mathrm{DMD}=0^{\mathrm{a}}$ \\
\hline & $\mathrm{h}$ & $0,001<\mathrm{DMD}<0,1$ & $0,001<\mathrm{DMD}<0,1$ & $\mathrm{DMD}=0^{\mathrm{a}}$ \\
\hline & $\mathrm{i}$ & $5<\mathrm{DMD}<10$ & $0,5<\mathrm{DMD}<1$ & $\mathrm{DMD}=0^{\mathrm{a}}$ \\
\hline \multirow{2}{*}{ REB } & $\mathrm{a}$ & $20<\mathrm{DMD}<50$ & $0,1<$ DMD $<0,5$ & $\mathrm{DMD}=0^{\mathrm{a}}$ \\
\hline & $\mathrm{b}$ & $10<\mathrm{DMD}<15$ & $0,1<\mathrm{DMD}<0,5$ & $\mathrm{DMD}=0^{\mathrm{a}}$ \\
\hline
\end{tabular}


OPTIMISATION DES PROGRAMMES DE SURVEILLANCE

\section{TABLEAU V}

Suite.

\begin{tabular}{|c|c|c|c|c|}
\hline \multirow{2}{*}{$\begin{array}{l}\text { Composition } \\
\text { isotopique }\end{array}$} & \multicolumn{4}{|c|}{ Dose minimale détectable avec un niveau de confiance de $95 \%(\mathrm{mSv})$} \\
\hline & Programme & $P(i)$ uniforme & $P(i)$ exponentielle & $P(i)$ alpha \\
\hline \multirow{7}{*}{ REB } & $\mathrm{c}$ & $1<\mathrm{DMD}<2$ & $0,01<\mathrm{DMD}<0,1$ & $\mathrm{DMD}=0^{\mathrm{a}}$ \\
\hline & $\mathrm{d}$ & $20<\mathrm{DMD}<50$ & $0,1<\mathrm{DMD}<0,5$ & $\mathrm{DMD}=0^{\mathrm{a}}$ \\
\hline & $\mathrm{e}$ & $1<\mathrm{DMD}<2$ & $0,01<\mathrm{DMD}<0,1$ & $\mathrm{DMD}=0^{\mathrm{a}}$ \\
\hline & $\mathrm{f}$ & $2<\mathrm{DMD}<3$ & $0,1<\mathrm{DMD}<0,5$ & $\mathrm{DMD}=0^{\mathrm{a}}$ \\
\hline & $\mathrm{g}$ & $0,1<\mathrm{DMD}<0,5$ & $0,001<\mathrm{DMD}<0,1$ & $\mathrm{DMD}=0^{\mathrm{a}}$ \\
\hline & $\mathrm{h}$ & $0,001<\mathrm{DMD}<0,1$ & $0,001<\mathrm{DMD}<0,1$ & $\mathrm{DMD}=0^{\mathrm{a}}$ \\
\hline & $\mathrm{i}$ & $1<\mathrm{DMD}<5$ & $0,5<\mathrm{DMD}<1$ & $\mathrm{DMD}=0^{\mathrm{a}}$ \\
\hline \multirow{9}{*}{ MOX } & a & $20<\mathrm{DMD}<50$ & $0,1<\mathrm{DMD}<0,5$ & $\mathrm{DMD}=0^{\mathrm{a}}$ \\
\hline & b & $10<\mathrm{DMD}<15$ & $0,1<\mathrm{DMD}<0,5$ & $\mathrm{DMD}=0^{\mathrm{a}}$ \\
\hline & $\mathrm{c}$ & $1<\mathrm{DMD}<2$ & $0,01<\mathrm{DMD}<0,1$ & $\mathrm{DMD}=0^{\mathrm{a}}$ \\
\hline & d & $20<\mathrm{DMD}<50$ & $0,1<\mathrm{DMD}<0,5$ & $\mathrm{DMD}=0^{\mathrm{a}}$ \\
\hline & $\mathrm{e}$ & $1<\mathrm{DMD}<2$ & $0,01<\mathrm{DMD}<0,1$ & $\mathrm{DMD}=0^{\mathrm{a}}$ \\
\hline & $\mathrm{f}$ & $2<\mathrm{DMD}<3$ & $0,1<\mathrm{DMD}<0,5$ & $\mathrm{DMD}=0^{\mathrm{a}}$ \\
\hline & $\mathrm{g}$ & $0,1<\mathrm{DMD}<0,5$ & $0,001<\mathrm{DMD}<0,1$ & $\mathrm{DMD}=0^{\mathrm{a}}$ \\
\hline & $\mathrm{h}$ & $0,001<\mathrm{DMD}<0,1$ & $0,001<\mathrm{DMD}<0,1$ & $\mathrm{DMD}=0^{\mathrm{a}}$ \\
\hline & $\mathrm{i}$ & $1<\mathrm{DMD}<5$ & $0,5<\mathrm{DMD}<1$ & $\mathrm{DMD}=0^{\mathrm{a}}$ \\
\hline \multirow{9}{*}{ MOX_RP } & $\mathrm{a}$ & $20<\mathrm{DMD}<50$ & $0,1<\mathrm{DMD}<0,5$ & $\mathrm{DMD}=0^{\mathrm{a}}$ \\
\hline & $\mathrm{b}$ & $10<\mathrm{DMD}<15$ & $0,1<\mathrm{DMD}<0,5$ & $\mathrm{DMD}=0^{\mathrm{a}}$ \\
\hline & $\mathrm{c}$ & $1<\mathrm{DMD}<2$ & $0,01<\mathrm{DMD}<0,1$ & $\mathrm{DMD}=0^{\mathrm{a}}$ \\
\hline & d & $20<\mathrm{DMD}<50$ & $0,1<\mathrm{DMD}<0,5$ & $\mathrm{DMD}=0^{\mathrm{a}}$ \\
\hline & $\mathrm{e}$ & $2<\mathrm{DMD}<3$ & $0,01<\mathrm{DMD}<0,1$ & $\mathrm{DMD}=0^{\mathrm{a}}$ \\
\hline & $\mathrm{f}$ & $3<\mathrm{DMD}<4$ & $0,01<\mathrm{DMD}<0,1$ & $\mathrm{DMD}=0^{\mathrm{a}}$ \\
\hline & $\mathrm{g}$ & $0,5<\mathrm{DMD}<1$ & $0,001<\mathrm{DMD}<0,1$ & $\mathrm{DMD}=0^{\mathrm{a}}$ \\
\hline & $\mathrm{h}$ & $0,5<\mathrm{DMD}<1$ & $0,001<\mathrm{DMD}<0,1$ & $\mathrm{DMD}=0^{\mathrm{a}}$ \\
\hline & $\mathrm{i}$ & $10<\mathrm{DMD}<20$ & $0,1<\mathrm{DMD}<0,5$ & $\mathrm{DMD}=0^{\mathrm{a}}$ \\
\hline
\end{tabular}

${ }^{\mathrm{a}} \mathrm{La}$ dose $0 \mathrm{mSv}$ correspond à un percentile supérieur au $95^{\mathrm{e}}$. 
Le programme en place (c) permet d'assurer la détection de doses comprises entre 1 et $2 \mathrm{mSv}$ pour toutes les provenances de combustible en supposant $P(i)$ uniforme. Pour $P(i)=e^{-i}$, la DMD est comprise entre 0,01 et $0,1 \mathrm{mSv}$. Ainsi, ce programme est un bon compromis entre le nombre de mesures et la sensibilité du programme de surveillance si les résultats obtenus sous les hypothèses des deux probabilités a priori de l'incorporation sont considérées. De plus, ce programme est simple à mettre en œuvre et peut convenir également à d'autres expositions par le plutonium, notamment lors du démantèlement d'installations, car il est raisonnablement prudent pour la surveillance de l'exposition à l'oxyde de plutonium. Cette étude conforte donc le choix du service de santé au travail d'utiliser ce programme pour ce groupe de travailleurs.

Passer d'une période des mesures urinaires de 6 mois à 3 mois induit une augmentation de la DMD avec la probabilité a priori exponentielle. Cette augmentation est un artefact causé par le fait qu'ajouter des mesures dont le résultat est compris entre zéro et le seuil de décision apporte une information qui contrebalance l'information apportée par la probabilité a priori.

\section{Développement d'OPSCI}

Le logiciel OPSCI (Optimisation du programme de surveillance de la contamination interne) a été développé afin d'aider à la mise en place et à l'évaluation d'un programme de surveillance. Il utilise les méthodes présentées ici. Il permet dans un premier module de calculer la dose efficace en appliquant les recommandations de la CIPR (ICRP, 1997).

Un second module estime, pour un programme de surveillance donné, la DMD en prenant en compte les incertitudes sur le calcul de dose. Pour cela, il utilise la méthode WeLMoS et la connaissance (incertaine) de l'utilisateur sur la mesure et sur les conditions d'exposition.

Le dernier module permet le calcul des DMD relatives aux différents programmes de surveillance que l'utilisateur veut évaluer. En prenant en considération ces DMD et le coût des analyses à effectuer, il pourra juger de la pertinence des programmes. Pour cela, le réseau bayésien est utilisé. Si nécessaire, l'utilisateur peut préciser la DMD du programme qu'il juge le plus pertinent avec le deuxième module.

OPSCI a été validé en comparant à chaque étape de son développement ses résultats avec la littérature scientifique et avec le logiciel IMBA (HPA, Puncher and Birchall, 2007). 


\section{Discussion, conclusions et perspectives}

Les méthodes qui ont été utilisées permettent de comparer la qualité des programmes de surveillance au moyen d'une grandeur que nous avons définie comme la DMD. Cette comparaison est évidemment à analyser en regard avec les contraintes de dose visées par le programme.

\subsection{Propagation des incertitudes}

Le choix entre l'utilisation de la méthode WeLMoS ou du réseau bayésien dépend notamment de l'information disponible sur les paramètres incertains. Une information conséquente et disponible permet de réduire fortement la subjectivité dans le choix du modèle probabiliste pour représenter les incertitudes sur les paramètres. La méthode WeLMoS est alors une méthode donnant rapidement une estimation très précise de la DMD. Cependant, si l'information disponible semble insuffisante pour justifier d'un choix particulier de modèle probabiliste, alors le réseau bayésien semble mieux adapté, car il permet d'apprécier directement l'impact de ce choix sur l'évaluation de la DMD. Il serait intéressant de comparer les résultats obtenus par ces deux méthodes avec ceux obtenus par l'UF code (LANL, Miller, 2008) utilisant un jeu de modèles biocinétiques et un algorithme de chaîne de Markov.

Enfin, une modélisation plus flexible et réaliste des incertitudes peut également être faite dans le cadre théorique des probabilités imprécises. Il serait donc intéressant d'intégrer cette modélisation notamment au sein du réseau bayésien établi.

\subsection{Probabilité a priori}

La probabilité a priori de l'incorporation est la vision subjective de l'utilisateur avant toute mesure. Cette subjectivité est inévitable mais elle peut être assise sur des données historiques (Miller et al., 1993) ou déterminée à partir d'un cas de contamination similaire en cas de suivi post-incidentelle (James et al., 2008).

Dans cette étude, la subjectivité du choix a été quantifiée en utilisant plusieurs probabilités a priori modélisant des visions extrêmes de la contamination potentielle. De telles approches sont nécessaires pour évaluer l'influence de la probabilité a priori sur le résultat.

De façon plus générale, la définition de la probabilité a priori de l'incorporation peut être améliorée en intégrant les mesures de la contamination d'ambiance 
des postes de travail réalisées par le service de protection radiologique et qui apportent une information complémentaire des analyses radiotoxicologiques.

\subsection{Conclusions}

Dans cette étude, pour la première fois, deux méthodes ont été utilisées, pour évaluer la DMD : la méthode WeLMoS ou un réseau bayésien attribuent des probabilités a priori à tous les paramètres incertains, dont l'incorporation, qui sont actualisées en probabilités a posteriori par la connaissance apportée par les analyses radiotoxicologiques.

Ces deux méthodes fournissent un cadre général pour estimer la DMD et la qualité d'un programme de surveillance en comparant la DMD avec une contrainte de dose. Cette méthodologie a été appliquée au programme de surveillance des travailleurs en charge de la purification du plutonium sur le centre AREVA NC de La Hague. Après une estimation des DMD correspondantes à différents programmes de surveillance envisageables par l'utilisation des méthodes développées, il a été conclu, sous des hypothèses explicites sur les probabilités a priori des paramètres incertains, que le programme de surveillance en place est le meilleure compromis entre les coûts des analyses et la sensibilité du programme.

Cette étude souligne l'intérêt de la DMD comme un outil pour optimiser les programmes de surveillance de la contamination interne en prenant en compte les incertitudes sur les conditions d'exposition et de mesure.

\subsection{Perspectives}

Les méthodes développées peuvent être appliquées à tout programme de surveillance. Elles seront prochainement utilisées pour étudier la surveillance de l'uranium, en s'intéressant aux questions de la possible toxicité rénale et de l'apport alimentaire en uranium naturel.

Elles pourraient également aider à la définition de programmes de surveillance adaptés pour les personnels soignants des services de médecine nucléaire. Cette problématique est très différente de la surveillance dans l'industrie du cycle nucléaire car les radionucléides manipulés ont des périodes très courtes rendant l'incertitude sur la date de contamination et la période de surveillance primordiales.

Remerciements. Ce projet a été financé dans le cadre du programme d'intérêt commun «DOSINTER » entre l'IRSN et AREVA. 


\section{RÉFÉRENCES}

Davesne E., Casanova P., Chojnacki E., Paquet F., Blanchardon E. (2010) Optimisation of internal contamination monitoring programme by integration of uncertainties, Radiat. Prot. Dosim. 30, $5-21$.

ICRP Publication 66 (1994) Human respiratory tract model for radiological protection, Ann. ICRP 24 (1-3).

ICRP Publication 75 (1997) General principles for the radiation protection of workers, Ann. ICRP 27 (1).

ICRP Publication 107 (1998) CD-ROM Version 2.01: The ICRP database of dose coefficients: workers and members of the public: An extension of ICRP publications 68 and 72.

ISO (2006) Radiation protection - Monitoring of workers occupationally exposed to a risk of internal contamination with radioactive material, ISO 20553:2006(E).

James A.C., Birchall A., Puncher M. (2008) Uncertainty in internal doses: using Bayes to transfer information from one worker to another. In: IRPA 12, 19-27 October 2008, Buenos Aires, Argentina.

Marsh J.W., Castellani C.M., Hurtgen C., Lopez M.A., Andrasi A., Bailey M.R., Birchall A., Blanchardon E., Desai A.D., Dorrian M.D., Doerfel H., Koukouliou V., Luciani A., Malatova I., Molokanov A., Puncher M., Vrba T. (2008) Internal dose assessments: uncertainty studies and update of ideas guidelines and databases within CONRAD project, Radiat. Prot. Dosim. 131, 34-39.

Miller G., Inkret W.C., Martz H. (1993) Bayesian detection analysis for radiation exposure, Radiat. Prot. Dosim. 48, 251-256.

Miller G., Inkret W.C., Little T.T., Martz H.F., Schillaci M.E. (2001) Bayesian prior probability distributions for internal dosimetry, Radiat. Prot. Dosim. 94, 347-352.

Miller G. (2008) Variability and uncertainty of biokinetic model parameters: the discrete empirical Bayes approximation, Radiat. Prot. Dosim. 131, 394-398.

Puncher M., Birchall A. (2007) Estimating uncertainty on internal dose assessments, Radiat. Prot. Dosim. 127, 544-547.

Puncher M., Birchall A. (2008) A Monte Carlo method for calculating Bayesian uncertainties in internal dosimetry, Radiat. Prot. Dosim. 132, 1-12.

Vivier A., Fottorino R., Rousse B. (2010) Seuil de décision et limite de détection : estimation, interprétation et optimisation. $1^{\text {re }}$ partie : les principes de base, Radioprotection 45 (3), 321-343. 